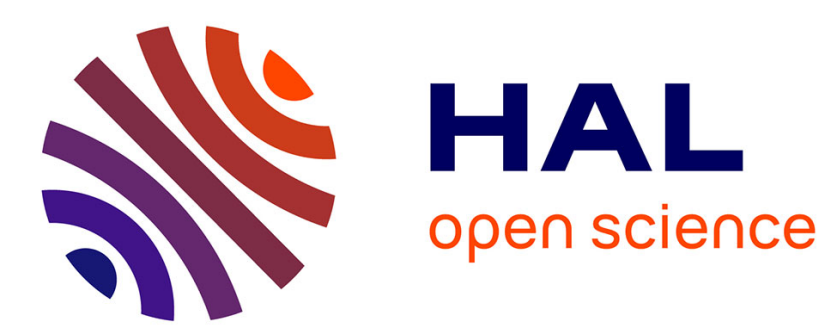

\title{
The puzzle of cooperation in a game of chicken: An experimental study
}

Marie-Laure Cabon-Dhersin, Nathalie Etchart-Vincent

\section{To cite this version:}

Marie-Laure Cabon-Dhersin, Nathalie Etchart-Vincent. The puzzle of cooperation in a game of chicken: An experimental study. Theory and Decision, 2012, 72 (1), pp.65-87. 10.1007/s11238010-9220-9 . hal-00636089

\section{HAL Id: hal-00636089 \\ https://hal.science/hal-00636089}

Submitted on 26 Oct 2011

HAL is a multi-disciplinary open access archive for the deposit and dissemination of scientific research documents, whether they are published or not. The documents may come from teaching and research institutions in France or abroad, or from public or private research centers.
L'archive ouverte pluridisciplinaire HAL, est destinée au dépôt et à la diffusion de documents scientifiques de niveau recherche, publiés ou non, émanant des établissements d'enseignement et de recherche français ou étrangers, des laboratoires publics ou privés. 


\title{
The Puzzle of Cooperation in a Game of Chicken: An Experimental Study
}

Marie-Laure Cabon-Dhersin*

\author{
Ecole Normale Supérieure de Cachan \\ $\&$
}

Centre d'Economie de la Sorbonne (CES).

61, Avenue du Président Wilson, 94230 Cachan, France

\section{Nathalie Etchart-Vincent}

\author{
Centre International de Recherche sur \\ l'Environnement et le Développement \\ (CIRED), \\ UMR 8568 CNRS/EHESS \\ Campus du Jardin Tropical, \\ 45 bis, Avenue de la Belle Gabrielle, \\ 94736 Nogent sur Marne Cedex, France
}

\begin{abstract}
The objective of the paper is to investigate the impact of agents' heterogeneity (as regards their attitude toward cooperation) and payoff structure on cooperative behaviour, using an experimental setting with incomplete information. A game of chicken is played considering two types of agents: 'unconditional cooperators', who always cooperate, and 'strategic cooperators', who do not cooperate unless it is in their interest to do so. Overall, our data show a much higher propensity to cooperate than predicted by theory. They also suggest that agent heterogeneity matters: the higher the proportion of 'strategic cooperators' in the population, the higher their probability to cooperate. Finally, our data confirm that higher rewards to cooperation (embedded in the payoff structure) tend to lower defection. Taken together, our results suggest that the subjects might be non-expected utility maximizers, dealing with both outcomes and probabilities in a non-linear manner.
\end{abstract}

Keywords: Social dilemma; Game of Chicken; Cooperation; Experiment; Heterogeneous population.

JEL classification: $\mathrm{C} 72$, C92

Corresponding author: Marie-Laure Cabon-Dhersin, Department of Social Sciences, Ecole Normale Supérieure de Cachan, 61, Avenue du Président Wilson, 94230 Cachan, France; Tel: (+33) 1474028 42; Email: cabon@ sociens.ens-cachan.fr. 


\section{The Puzzle of Cooperation in a Game of Chicken:}

An Experimental Study

\section{Introduction}

The puzzle of cooperation has attracted and continues to attract social scientists seeking to explain why, in real life, many people tend to exhibit cooperative patterns of behaviour despite strong incentives to free-ride. Indeed, in the absence of any monetary incentive, why do individuals choose to cooperate, when they may be betrayed by their partner?

During the last decade, there has been a surge of experimental research devoted to the investigation of individuals' propensity to cooperate in one-shot interactions. A variety of two- and multiple-person games (prisoner's dilemma game, trust game, public good game or game of chicken) have been studied to capture 'social dilemma' situations, in which selfinterest behaviour leads to an outcome that is worse for everyone concerned (Hardin 1971; Baron 2008). A common result of these diverse experiments is one major and recurrent phenomenon: the level of cooperation observed is much higher than predicted by theory (see, among many others, Berg et al. 1995; Güth et al. 1997).

Several kinds of theoretical explanations have been proposed and empirically investigated to resolve the puzzle of cooperation, ranging from biological to ecological, anthropological and social features of evolution (Ridley 1997). In this respect, social identityrelated factors (Dawes et al. 1988; Kollock 1998b; Yamagishi and Kiyonari 2000; Wit and Wilke 1992), social norms (Thogersen 2008), as well as socio-demographic and cultural factors (Boone and van Witteloostuijn 1999; Gächter et al. 2004) have been found to play a role in the prevalence of cooperative behaviour. In the same vein, personality ${ }^{1}$ can be shown to strongly affect behaviour in interactive settings, with aggressive people being less cooperative than sociable ones (Boone et al. 1999).

Cooperation is also induced when cheating generates psychological costs that individuals want to avoid. More generally, some agents appear to be motivated by considerations of "fairness" and "reciprocity" that may make them opt for cooperation (Bolton and Ockenfels 2000; Charness and Rabin 2002; Dawes and Thaler 1988; Falk and

\footnotetext{
${ }^{1}$ In that study, personality was captured through such indicators as sensations seeking or locus of control.
} 
Fischbacher 2006; Fehr and Schmidt 1999; Levine 1998; Rabin 1993). Finally, cognitive limitations may lead some subjects to use in one-shot games the rules of thumb they have developed in repeated games (Goeree and Holt 2001), which may increase cooperation in experimental settings.

While the above-mentioned arguments make an important contribution to explaining why people cooperate much more than they are expected to, the present paper attempts to extend these findings along two hitherto less explored dimensions. Herein lies the original contribution of our work.

The first point concerns the specific nature of the game involved in our experimental study, namely a 'Game of Chicken'. Strangely enough, while the 'Prisoner's Dilemma Game' (PDG), emblematic of Pareto-inferior outcomes that result from non-cooperation, has been extensively studied, the game of chicken, representing a social dilemma issuing from noncoordination, has been largely neglected by experimentalists. Thus, by focussing on the game of chicken, we push the frontiers of our understanding of social dilemmas further.

As in the (PDG), in the game of chicken, each partner appears to benefit more from bilateral cooperation than from bilateral defection. But, contrary to what happens in the PDG, if the agent expects her partner to defect, she will have interest to cooperate, and she will be declared the "chicken" of the game. Therefore, in the game of chicken, two pure equilibria exist (corresponding to unilateral cooperation and unilateral defection respectively), with no dominating strategy. The game of chicken thus appears to be a realistic description of strategic interactions, which is particularly suitable for describing relations between individuals, firms, institutions, social groups, political parties and countries that wish to coordinate for mutual benefit. It has also been used to describe military or political conflict (Snyder 1971; Stone 2001), as well as negotiations regarding environmental conventions (Carraro and Siniscalco 1993; Ward 1993).

The second point has to do with agent heterogeneity, a fact of real-life that most models in economic theory, and particularly in game theory, assume away. This is however changing as evidence mounts from experiments confirming that agent homogeneity is the exception rather than being the rule. For instance, some recent and stimulating empirical results on cooperation suggest that, even though a non negligible minority or even a majority of subjects exhibit self-interest maximizing preferences, a significant proportion also exhibit other-regarding preferences (Erlei 2008). Furthermore, using data from a wide range of experiments, Fehr and Gächter (2000) (cited in Camerer 2003) estimate that 40 to $66 \%$ of the subjects show a preference for reciprocity, while only 20 to $30 \%$ of them care about their sole 
monetary gains (the remaining subjects do not seem to have very clearly defined preferences). A recent experimental study involving a Game of Chicken (Neugebauer et al. 2008) suggests that, even though most of the subjects $(83 \%)$ tend to behave in a self-interested way and to maximize their monetary gains, some of them appear to favour reciprocity and equity.

Though experimental results have confirmed the over-whelming presence of agent heterogeneity, they have not attempted to measure its consequences in strategic settings. In contrast, the objective of the present paper is precisely to investigate the impact of 'agent heterogeneity' on cooperative behaviour in a game of chicken, using a context-free experimental setting with incomplete information. To that purpose, we use the theoretical framework proposed by Cabon-Dhersin and Ramani (2007) (CDR 2007 in the following). The original feature of the CDR model is that it involves a population with two types of agents, namely the 'payoff maximizers', or 'strategic cooperators' (who do not cooperate unless it is in their interest to do so), and the 'unconditional cooperators' (who always choose to cooperate $^{2}$ ). Under the assumption of heterogeneity (in terms of preferences), the uncertainty as regards the partner's behaviour is twofold: endogenous uncertainty - as regards the action that could be chosen by the partner - strengthened by the uncertainty as regards her type (since her behaviour now also depends on her type). Then it examines (among other things) how the level of cooperation is affected by the heterogeneity of the population, i.e. by the probability that one faces a partner with different preferences.

The above-mentioned framework allows us to examine how, and to what extent, the structure of the population and the structure of the payoffs are likely to affect cooperative behaviour. Our experimental design was formulated to identify each subject's type before investigating the following four predictions of the CDR model:

1. The higher the proportion of 'strategic cooperators' in the population (or, in other words, the higher the probability to meet and play against a 'strategic cooperator'), the higher their probability to cooperate.

2. A minimum proportion of 'strategic cooperators' in the population is required to induce a cooperative behaviour on their part.

3. Beyond the same threshold (i.e. beyond the same given proportion of 'strategic cooperators' in the population), the level of cooperation in the whole population no longer depends on the proportion of 'strategic cooperators', but is only affected by the structure of the payoffs.

\footnotetext{
2 Their actions are driven by some other motives than opportunism (e.g. self respect, altruism, ethical considerations).
} 
4. The higher the unilateral defection (resp. unilateral cooperation) gain, the lower (resp. higher) the level of cooperation among the 'strategic cooperators'.

The central results of our experiment can be summarized as follows. Our experimental data show individuals to have a much higher propensity to cooperate than predicted: the observed proportion of cooperative choices turns out to be systematically 20 to 50 points higher than theoretically expected. The data also suggest that agent heterogeneity matters: the higher the proportion of 'strategic cooperators' in the population, the higher their probability to cooperate. Finally, our experiments confirm that higher rewards to cooperation (embedded in the payoff structure) tend to lower defection.

The present experimental results offer some interesting prospects for the extension of game-theoretic models concerned with both agent heterogeneity and cooperation. In particular, they suggest that some of the gap between predicted and observed cooperative behaviour might be due to the underweighting/overweighting of the probability of partnering with a player of a specific type. In this respect, it would be worth considering that the subjects might be non-expected utility maximizers, dealing with both outcomes and probabilities in a non-linear manner, as 'Rank Dependent Utility' or 'Prospect Theory' for instance seem to suggest. In other words, it would be worth incorporating more sophisticated risk preferences into models with agent heterogeneity, in order to allow the impact of non-linear probability weighting on cooperative behaviour to be investigated in a more systematic manner.

The theoretical framework and the predictions that our experimental study was designed to test are presented in more detail in section 2. Section 3 describes the experimental design and procedure. Sections 4 and 5 are devoted to the results and discussion respectively. Section 6 concludes.

\section{The theoretical framework and predictions}

CDR (2007) is based on a two-person two-stage game of chicken with incomplete information. For the sake of simplicity, and with no loss of generality, only the one-stage version of the model will be presented here ${ }^{3}$. As said in the introduction, the population of the players is assumed to consist of two kinds of agents, called 'strategic cooperators' (denoted

\footnotetext{
${ }^{3}$ The pilot experiment was run using the two-stage version of the game, involving a first choice between entry and non entry, and, in case of entry, a second choice between cooperation and non cooperation. The resulting choice situations appeared to be too complex for the subjects, resulting in poor concentration and unreliable answers.
} 
SCs in the following) and 'unconditional cooperators' (denoted UCs) respectively. The SCs are expected-value maximizers and choose not to cooperate unless it pays to do so. Consequently, the SCs can either defect, choosing action $d$, or cooperate, opting for action $c$. By contrast, the UCs follow the simple rule always to cooperate, whether it pays to do so or not. Their only option is thus to choose cooperation and play $c$.

In the game, each player knows her own type and has some probabilistic information about the type of her partner. Let $p \in(0,1)$ be the probability that the player's partner is a SC. This probability is exogenously given and is common knowledge to all players. Notice that $p$ can also be interpreted as the proportion of SCs in the population. Besides, each player expresses a belief as regards the behaviour of her fellow player. The game is symmetrical, in the sense that the structure of the payoffs and the beliefs of players of the same type are assumed to be identical. For each player, her endogenous belief that her fellow player will not cooperate if she is a $S C$ is given by a probability $\alpha$ and it lies in the interval $(0,1)$. So, her belief that her partner (whatever her type) will cooperate is given by the probability $(1-p \alpha)^{4}$.

The payoff structure of the game is as follows. Each player wins $X$ if both players cooperate and $Y$ if they both deviate. If a SC defects while her partner cooperates, she gets $H$ $>X$ while her partner gets $L>Y$ with $L<X$. This payoff structure clearly captures the essence of the Game of Chicken. The matrix of the game is given in Figure 1.

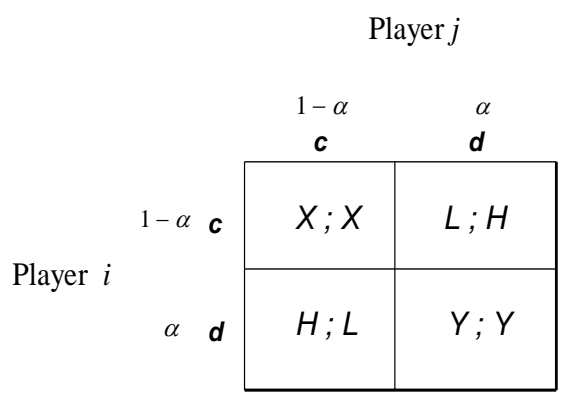

Figure 1. A typical matrix of the Game of Chicken $(H>X>L>Y)$.

\footnotetext{
${ }^{4}$ This probability corresponds to the probability $1-p$ to meet a UC (who always cooperates) added to the probability $(1-\alpha) p$ to meet a SC who chooses to cooperate.
} 
The game of chicken allows multiple equilibria. Since defection is not a dominant strategy, it is possible that, at least in some equilibria, cooperation prevails even among the $\mathrm{SCs}^{5}$. Here, we will focus on those situations in which cooperation can be observed, i.e. in which the SCs will choose to cooperate with a positive probability $(1-\alpha)>0$. Besides, since the structure of the payoffs and the beliefs of players of the same type are assumed to be identical, only symmetrical equilibria will be considered.

For a SC, a necessary condition for cooperation is that her expected returns from cooperation be greater than those from defection. This condition is given by:

$$
p[(1-\alpha) X+\alpha L]+(1-p) X>p[(1-\alpha) H+\alpha Y]+(1-p) H
$$

In other words, we have:

$$
(1-p \alpha)<T, \text { with } T=\frac{L-Y}{(H-X)+(L-Y)}
$$

Some remarks should be made at this stage. First, in a game of chicken, cooperation is possible but never certain. In fact, it can be shown that the exact probability with which a SC will defect is given by $\alpha=\frac{1-T}{p}$. Second, a necessary condition for cooperation to emerge is that the probability of non-defection (i.e. the probability that the partner chooses cooperation), given by $(1-p \alpha)$, be not too high. When $(1-p \alpha)$ is equal or superior to a given value $T$, i.e. when $(1-p) \geq T$ (with $\alpha<1$ ), it is always optimal for a SC to deviate (The details of the proofs are given in CDR, 2007).

Result: In the one-shot one-stage game of chicken under consideration, the symmetrical Nash equilibrium is such that:

(i) Whenever $p>(1-T)$, SCs will cooperate with a probability $(1-\alpha)$ such that:

$$
(1-\alpha)=1-\frac{1-T}{p}=1-\frac{1}{p} \frac{H-X}{(H-X)+(L-Y)} .
$$

(ii) Whenever $p \leq(1-T)$, SCs will always defect $(\alpha=1)$.

\footnotetext{
${ }^{5}$ It is a well-known result in standard game theory (when all players are assumed to be 'self-interested') that any Game of Chicken offers three equilibria, two of them involving pure strategies. In this case, one of the players initiates a conflict and the other one chooses to cooperate, leading the latter to be declared the «chicken » of the game. The last equilibrium involves completely mixed strategies, with each player assigning a positive probability to each of her strategies, so as to maximize her payoffs. So, $(1-\alpha)=L / L+H$ corresponds to the mixed-strategy equilibrium of the game when there is no heterogeneity in the population, i.e. when all the players are assumed to be 'self-interested'.
} 
Remembering that the a priori probability $p$ that the partner be a SC may also be interpreted as the proportion of SCs in the population, the following four testable predictions P1 to P4 can be drawn from the previous result.

P1: A minimum proportion (1-T) of SCs in the population is necessary to induce a cooperative behaviour on their part.

When the population contains only a few SCs, these will always defect because they know that their partner is very likely to cooperate (see Figure 2, left part).

P2: When $p>(1-T)$, the higher the proportion $p$ of SCs in the population, the stronger their incentive to cooperate.

When $p>(1-T), \alpha=\frac{(1-T)}{p}$ at equilibrium. So, the probability $\alpha$ assigned by a SC to defection decreases when $p$ increases (see Figure 2, right part). Note that, for $p=1, \alpha=1-T$. This corresponds to the standard mixed-strategy equilibrium when there is no heterogeneity in the population, i.e. when the population contains only expected-value maximizers (SCs).

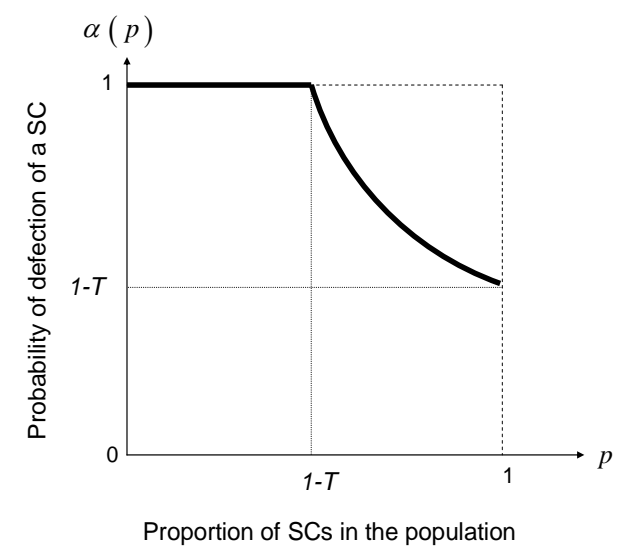

Figure 2. Predicted probability of defection $\alpha$ depending on the proportion of SCs in the population (with $H=120, L=70, X=100$ and $Y=50$ ).

P3: For any given Game of Chicken structure of payoffs and for any population with $p>(1-T)$, the proportion of cooperative agents in the whole population is constant and equal to $T$. 
As long as the proportion $p$ of SCs remains lower than $(1-T)$, the expected return from defection remains higher than that from cooperation, and any SC must defect whatever the game of chicken payoff structure $(\alpha=1)$. Therefore, the proportion of agents who cooperate in the whole population is equal to the proportion $(1-p)$ of UCs in the population (see Figure 3 , left part).

When $p$ becomes higher than $(1-T)$, the probability of defection (given by the probability $\alpha$ at equilibrium), decreases as $p$ increases. Quite remarkably, the exogenous increase in the proportion $p$ of SCs is exactly counterbalanced by the decrease in their endogenous propensity to defect $\alpha$, so that the proportion $p \alpha$ of agents who defect in the whole population becomes constant and equal to $1-T$. Alternately, the proportion $(1-p \alpha)$ of agents who cooperate in the whole population is constant and equal to $T$ (see Figure 3, right part).

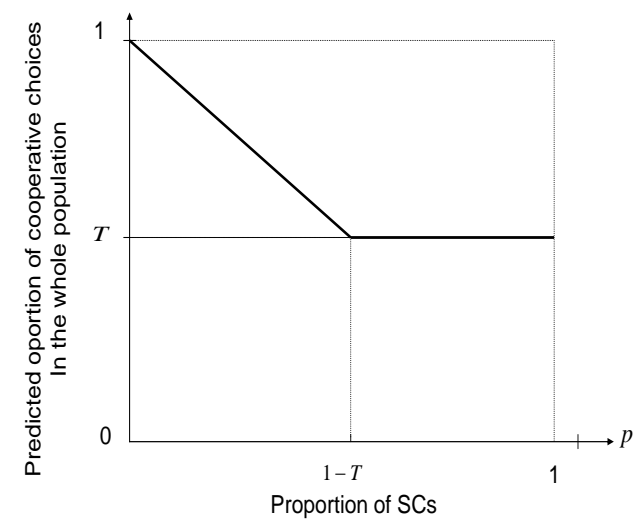

Figure 3. Predicted proportion of cooperative choices in the whole population depending on the proportion of SCs in the population

(with $H=120, L=70, X=100$ and $Y=50$ ).

P4a: The probability of defection $\alpha$ is an increasing function of the return from unilateral defection $(H)$.

P4b: The probability of defection $\alpha$ is a decreasing function of the return from unilateral cooperation $(L)$. $T=\frac{L-Y}{(H-X)+(L-Y)}$ is a decreasing function of $H$ and an increasing function of $L$. Since $\alpha=\frac{(1-T)}{p}, \alpha$ appears to be a decreasing function of $T$, thus an increasing function of $H$ and a decreasing function of $L$. 
Now, let us present the design of our experimental study, which aimed at testing the previous four predictions $\mathrm{P} 1$ to $\mathrm{P} 4$.

\section{The experimental design}

The final experiment included 85 subjects of which 45 were female. All the subjects were between 22 and 28 years old. Most of them were undergraduate students in Economics; the remaining ones were undergraduates in Mathematics. All of them were aware of game theory and decision theory, but with no specific skills in these topics.

The experiment was run during January and February 2007, and consisted in three successive and at least 15-days spaced independent sessions. In each session, the subjects' task consisted in the filling out of a paper-and-pencil questionnaire. Only the results from the first session/questionnaire will be reported here.

The questionnaire consisted of three separate parts, including several interactive choice situations each. In each choice situation, the subject was given a specific Game of Chicken payoff structure (with $X, Y, H$ and $L$ as in Figure 1 supra) as well as some probabilistic information about the type of her partner (being either a $\mathrm{SC}$ with a probability $p$ or a UC with a probability $(1-p))$ and asked to decide whether to cooperate or not with her. $X$ (bilateral cooperation gain) and $Y$ (bilateral defection gain) were given the same values $X=$ $100 €$ and $Y=50 €$ throughout the questionnaire.

The first part of the questionnaire aimed at investigating whether the subjects' behaviour changes as the proportion of SCs in the population increases from 0 to 1 (or, alternately, as the probability to meet a SC grows from 0 to 1), with the unilateral defection gain $H$ and the unilateral cooperation gain $L$ being kept constant and given the values $H=120$ $€$ and $L=70 €$ respectively (predictions P1, P2, P3). The second part of the questionnaire was designed to investigate whether the subjects' behaviour changes as the value of the unilateral defection gain $H$ increases from $100 €$ to $190 €$, with $p=0.75$ and $L$ being kept constant and given the value $L=70 €$ (Prediction P4a) Finally, the third part of the questionnaire aimed at investigating whether the subjects' behaviour changes as the value of the unilateral cooperation gain $L$ increases from $50 €$ to $95 €$, with $p=0.75$ and $H$ being kept constant and given the value $H=160 €$ (prediction P4b). 
To facilitate the subject's task, each part of the folder included a series of 3 questions which was built following the same pattern. A typical choice situation (drawn from the first part of the questionnaire) is reproduced in appendix A. In the $1^{\text {st }}$ and $2^{\text {nd }}$ questions, the subject was provided with the extreme values under investigation $(p=0$ and $p=1 ; H=100 €$ and $H=$ $190 € ; L=50 €$ and $L=95 €$ respectively). In the $3^{\text {rd }}$ question, a table recapitulated the whole range of 10 or 11 values $^{6}$; for each value in the table, the subject had to choose between cooperation and non cooperation by tipping the appropriate box. Note that the cooperative/non cooperative options were actually neutrally labelled 'red'/'blue', while the UCs (resp. SCs) were labeled 'type (a) agents' (resp. 'type (b) agents') to keep the wording as neutral as possible and avoid any framing effects, due to social and/or moral considerations for instance ${ }^{7}$.

The folder took about 15 minutes to be completed. The subjects were given written instructions, which were complemented with some oral instructions. In particular, the subjects were reminded that their partner would change from one question to another and that they had to consider each successive choice in isolation, especially when filling in the tables ${ }^{8}$. Besides, they were reminded that there were neither wrong nor right answers, and that they could switch from 'red' to 'blue' (or the opposite) as often as they wished to, or even choose to always play 'red' or always play 'blue'.

The participants were paid 5 euros for participating in the whole study (they were paid once they had completed the third questionnaire). Moreover, to help them consider their decisions as real despite the apparently hypothetical nature of the choice situations, a highincentive performance-based payment procedure was also introduced in each session ${ }^{9}$. At the beginning of the session, the participants were informed that i) two of them would be selected at random at the end of the session ${ }^{10}$, ii) each of them would be asked to draw (at random) one of the choice situations included in the questionnaire and to play it out for real against a real

\footnotetext{
${ }^{6}$ The first two questions actually provided guidance for the subjects. They were introduced after the pilot experiment showed that it was easier for the subjects to think of extreme values (which often induce simple decisions, due to dominance effects for instance) first.

${ }^{7}$ Indeed, even if only the 'deep structure' of the game is theoretically relevant for decision-making, there is a huge body of evidence confirming that the 'surface structure' (framing, wording, etc.) sharply influences the way people behave (Poppe, 2005; Wagenaar et al., 1988). In this respect, a socially-oriented framing might prevent a proper testing of theoretical predictions and confuse the data.

${ }^{8}$ Tables offer the advantage of simplicity and compacity, but they may also encourage the use of undesirable heuristics.

${ }_{9}$ Actually, since the sessions were completely independent from each other, each of them had its own performance-based payment procedure.

${ }^{10}$ Even though the ex ante probability of gain is quite low, the subjects appeared to be very sensitive to the payment procedure: they focused on the best outcome rather than on the probability of winning.
} 
partner (namely another participant), iii) her gains would depend on her previous choice as well as on her partner's previous choice. More details as regards the payment procedure are given in appendix $\mathrm{B}$.

Note that predictions P1 to P4 could not be tested unless the subjects' type is identified. In our experiment, each subject was classified through her behaviour in a single choice situation, namely the one encapsulated in question 1.2. ("You have 100\% chances to meet a partner of type (a), who always plays 'red'. Which colour do you choose?"; see appendix A). Indeed, this choice situation is the only one in which any self-interested subject is required to defect (play 'blue'). So, those subjects who chose to cooperate were labelled as non SCs, and the defective ones as SCs (see figure 4). Naturally, this rather weak criterion does not ensure that those latter subjects were genuine SCs. But the main point is that their behaviour throughout the questionnaire was fully compatible with that of a SC. Besides, and quite reassuringly, the rate of subjects labelled as SCs using our criterion appears to be very close to that found in Neugebauer et al. (2008)'s study involving a game of chicken $(86,7 \%$ versus $83 \%)$.

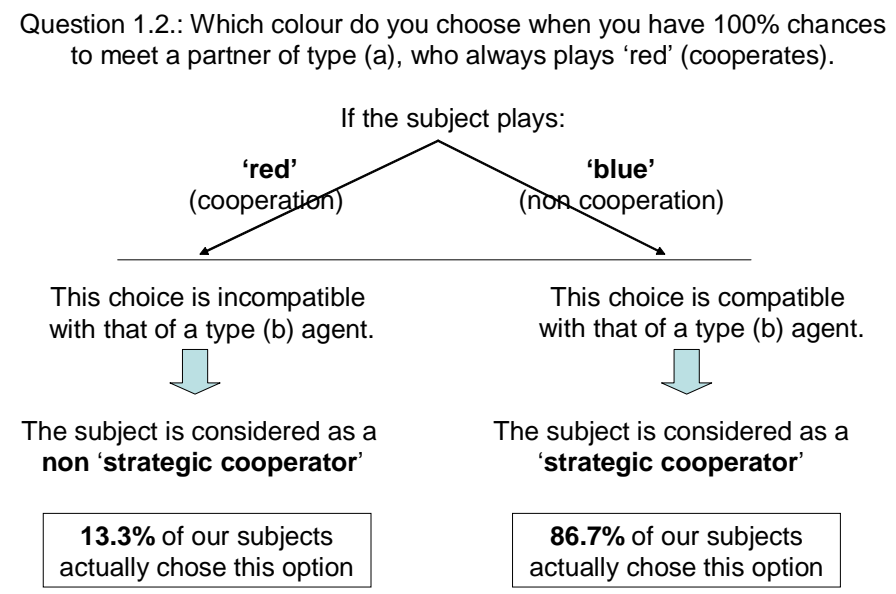

Figure 4. The identification of each subject's type using question 1.2.

It is important to note that those subjects who chose to cooperate in Choice Situation 1.2. were not classified as UCs, but only as non SCs. To understand why, the argument is twofold. First, an important point to make is that we did not need to collect any empirical information about the UCs to be able to test predictions P1-P4. Indeed, the behaviour of a UC is unambiguous since she always cooperates. So, given the proportion $(1-p)$ of UCs in the population (provided in each scenario of the questionnaire), the proportion of cooperative choices among the UCs could be obtained without considering the empirical population of 
UCs (it is simply equal to $(1-p)$ ). Second, we are well aware that the a priori SC and UC two categories are unlikely to exhaust the components of real world population. By the way, most of the subjects who chose to cooperate in choice situation 1.2. were not genuine UCs, since they chose not to cooperate in some other choice situations. So it would have been fallacious to label them as UCs.

This basic ambiguity, as well as the fact that we were not really interested in the UCs, led us to exclude from the set of data that was used to investigate the descriptive accuracy of the model all the subjects whose behaviour was not fully compatible with the SC profile ${ }^{11}$. After discarding those subjects who appeared to produce erratic answers, we were finally left with a 72 subject sample.

\section{Results}

We successively examine the impact on cooperation of population heterogeneity (predictions P1 to P3) and payoff structure (predictions P4a and P4b).

\subsection{Cooperation depending on the structure of the population}

We successively discuss the impact of population heterogeneity on cooperation among the SCs (P1 and P2) and among the whole population (P3).

First, note that, thanks to the law of large numbers, the probability $\alpha$ that a SC deviates (resp. the probability $(1-\alpha)$ that a SC cooperates) can be taken as the proportion of non cooperative choices (resp. cooperative choices) among the real SC population.

Now, graph 1 reports both the predicted and observed proportions of defective choices among SCs, depending on the hypothetical proportion of SCs in the population (given in the scenario). A proportion test shows that, for any strictly positive $p$, the difference between the predicted and observed levels of cooperation is always very significant and positive (every pvalue is equal or inferior to 0.001). Moreover, the degree of over-cooperation increases until $p$ $=0.75$. To better describe the results, we now distinguish the low and high parts of the curves.

For any $p \leq(1-T)=0.5, \mathbf{P 1}$ predicts that the SCs should not cooperate at all. For $p=$ 0 , all the SCs actually defect (as a result of the criterion used to identify the SCs in our

\footnotetext{
${ }^{11}$ Of course, non-SC subjects were not excluded from the performance-based payment procedure. Neither were the subjects who produced erratic answers.
} 
population). But when $p$ grows from 0 to 0.5 , the gap between the predicted and observed levels of cooperation grows, reaching almost 40 points for $p=0.5$.

When $p>(1-T)=0.5$, the level of cooperation grows as $p$ increases (i.e. as the probability of playing against a SC increases), as predicted by P2. And the theoretical and empirical curves appear to be remarkably parallel, which suggests that the model works qualitatively well when the SCs are in a majority in the population $(p>0.5)$. Nevertheless, the initial gap between the predicted and observed levels of cooperation remains, and is kept rather constant for $0.5 \leq p \leq 1$. It is noteworthy that, in the standard case without heterogeneity (i.e. for $p=1$ ), the level of cooperation is 20 points higher than expected: while no more than half cooperative choices should be observed among the SCs, this rate actually reaches 0.7 .

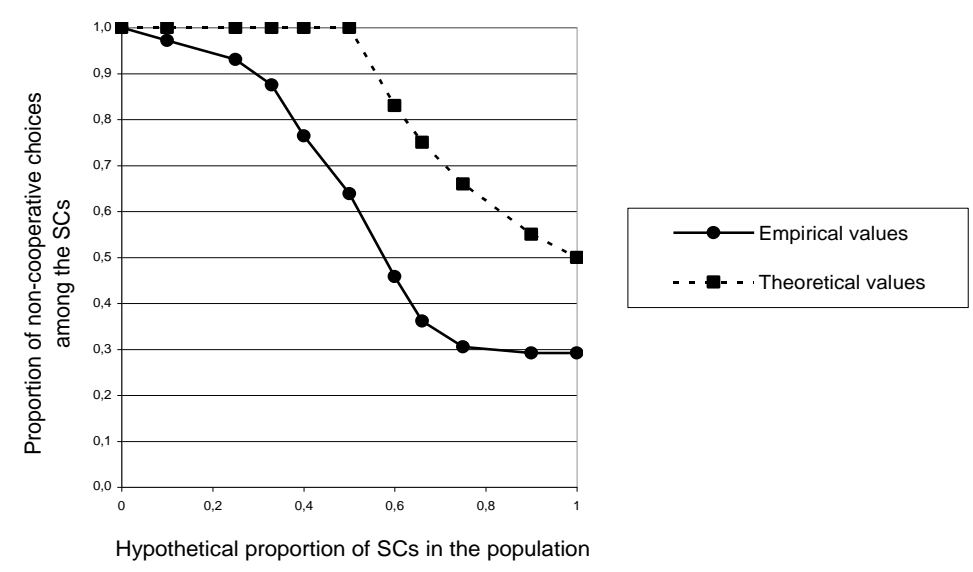

Graph 1. Predicted and observed proportion of non-cooperative choices among the SCs depending on the hypothetical proportion of SCs in the population.

Now, as regards P3, note that the proportion of cooperative choices in the whole population, given by $p(1-\alpha)+(1-p)$, was computed using the observed proportion $(1-\alpha)$ of cooperative choices among the SCs (identified as such using Question 1.2.), the hypothetical proportion $p$ of SCs in the population given in each scenario, and the hypothetical complementary proportion $(1-p)$ of UCs.

P3 predicts that the proportion of cooperative choices in the whole population depending on the proportion of SCs in the population should decrease from 1 to 0.5 as $p$ 
grows from 0 to 0.5 (see graph 2, theoretical values). Then, this proportion should remain constant, the increase in the proportion of cooperative SCs being exactly counterbalanced by the decrease in the proportion of UCs (who always cooperate). Though not completely supported by our data from a quantitative point of view, this strong theoretical property appears to be qualitatively rather satisfied.

As regards the left part of graph 2 (from $p=0$ to $p=0.25$ ), the theoretical and empirical curves match very well. Looking at graph 1 may help understand why. Actually, our SCs do cooperate more than expected (remember they should not cooperate at all), but since they are in a small minority in the population, their behaviour has not much impact on global behaviour $^{12}$.

Now, from $p=0.33$ to $p=0.5$, cooperation decreases slower than it should, so that the global level of cooperation remains 20 points too high. Once again, the explanation can be found in graph 1. Indeed, the degree of over-cooperation among the SCs grows with $p$. Since their weight in the population increases, the degree of over-cooperation among the whole population also increases.

From $p=0.5$ to $p=1$, the level of cooperation remains too high (it varies between 0.7 and 0.8 instead of remaining constant at 0.5). Moreover, the empirical curve exhibits a somewhat inverse- $U$ shape instead of a flat shape, which is due to the fact that the degree of over-cooperation among the SCs tends to decrease as $p$ grows. Since their weight in the population increases, the aggregate level of over-cooperation is also affected downward.

Anyway, a striking and noteworthy result is that the level of cooperation observed for $p=1$ is equal to its value for $p=0.5$, which fits the theoretical prediction and suggests that the difference between predicted and observed behaviour is essentially quantitative: on the one hand, the level of cooperation does not dramatically decrease as it should, and it always exceeds the predicted level by about 20 points; but, on the other hand, the empirical curve appears to be quite remarkably parallel to the theoretical one.

\footnotetext{
${ }^{12}$ Global behaviour is actually almost entirely driven by the UCs' deterministic behaviour.
} 


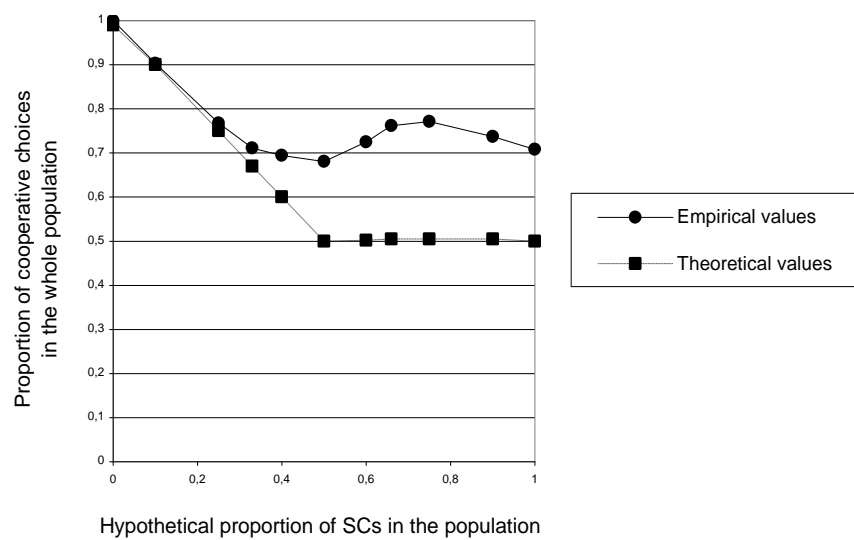

Graph 2. Predicted and empirical proportion of cooperative choices in the whole population depending on the hypothetical proportion of SCs in the population. 


\subsection{Cooperation depending on the structure of the payoffs}

The level of cooperation has been shown experimentally to depend on the structure of the payoffs (see for instance Sherman, 1969 or Güth et al., 1997). CDR (2007) predicts how the structure of the payoffs should affect behaviour (predictions P4a and P4b). Our experimental study precisely allows us to investigate $\mathrm{P} 4 \mathrm{a}$ and $\mathrm{P} 4 \mathrm{~b}$ and to identify the role played by $H$, the unilateral defection gain, and by $L$, the unilateral cooperation gain.

First, the level of cooperation is expected to decrease as $H$ grows (P4a), with a maximum rate of cooperative choices of 1 for $H=100 €$, and a flat right part and no cooperation at all when $H$ becomes superior or equal to $160 €$.

The level of cooperation actually appears to decrease as $H$ grows. Once again, the observed level of cooperation appears to be quantitatively higher than expected by 30 to 50 points, and this difference in level is highly significant for all $H$ (z test; every p-value is equal or inferior to 0.001$)$. But, on the other hand, the empirical curve appears to be qualitatively similar to the theoretical one (see Graph 3).

Quite remarkably, the rates of cooperative choices obtained for the last 4 values $(H=$ $160 €, 170 €, 180 €$, and $190 €$ ) appear not to be significantly different (proportion test, pvalue: 0.754$)$. The level of cooperation can thus be considered as constant between $H=160 €$ and $H=190 €$, which fits the theoretical prediction. However, the cooperation curve is still strongly translated upward: while for any $H \geq 160 €$, theory predicts that the SCs should no longer cooperate, the level of cooperation actually remains strongly positive (about $0.25 / 0.3$ ) even for the very deterrent gain of $190 €$.
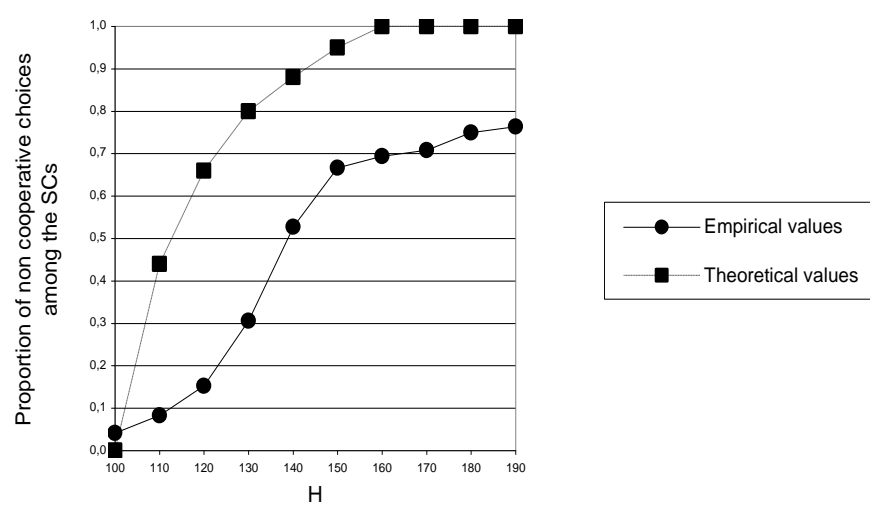

Graph 3. Predicted and empirical proportion of non cooperative choices among the SCs depending on unilateral defection gain $\mathrm{H}$. 
Now, the level of cooperation is expected to increase as $L$ grows $(\mathbf{P 4 b})$, with a flat left part and no cooperation at all as long as $L$ is inferior or equal to $70 €$, and a maximum rate of cooperative choices of about 0.25 for $L=95 €$ (see Graph 4).

The level of cooperation actually appears to increase as $L$ grows. However, as previously, the observed level of cooperation appears to be much higher than predicted (with all p-values equal or inferior to 0.001). Three striking features are worth noticing. First, the left part of the empirical curve $(L \leq 60 €)$ is flat, as predicted by the model. There is naturally a quantitative difference, since some cooperation exists while there should be no cooperation at all. But it seems that, as expected, proneness to cooperation is not sensitive to variations of $L$ when $L$ is kept small. As a second important feature, the rate of cooperative choices dramatically increases when $L$ grows from 60 to $80 €$, as if the subjects became highly sensitive to variations of $L$ when $L$ is given intermediate values, with a gap reaching 50 points for $L=80 €$. Thirdly, and contrary to theoretical predictions, the right part of the empirical curve appears to be flat, as if the subjects became insensitive to variations of $L$ when $L$ is given high values (from 80 to $95 €$ ). A proportion test run on the rate of non cooperative choices for these 4 values gives a p-value of 0.964 .

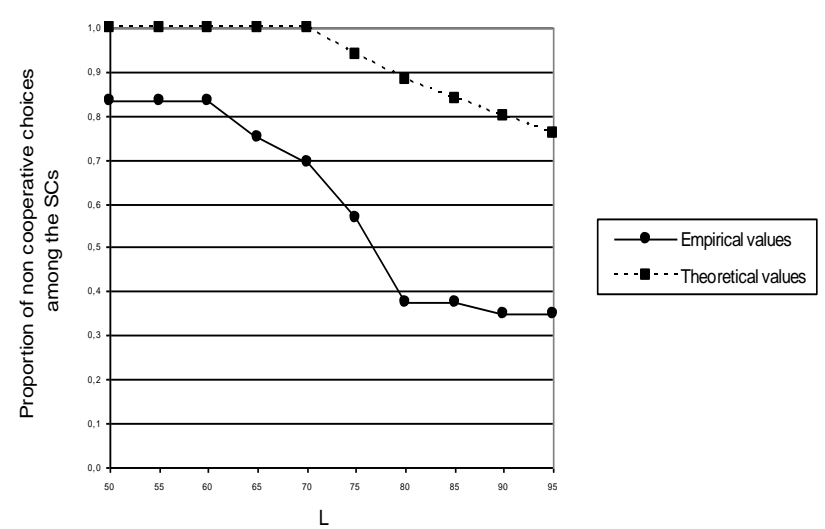

Graph 4. Predicted and observed proportion of non cooperative choices among the SCs depending on unilateral cooperation gain $\mathrm{L}$. 


\section{Discussion}

\subsection{Methodological discussion: the experimental design}

Part of the gap between the predicted and observed levels of cooperation might be explained by the fact that it was actually impossible to fully replicate experimentally the conditions under which the theoretical predictions were obtained. To be specific, two main assumptions had to be made as regards the behaviour of real subjects when designing the experiment to allow for the testing of the model. Since it is actually impossible to know whether these assumptions were experimentally satisfied or not, they might be viewed as undesirable 'auxiliary hypotheses', raising a Duhem-Quine argument and preventing us from drawing clear-cut conclusions from our data (see for instance Starmer, 1999). In the following paragraph, we will present these two assumptions, as well as some empirical arguments allowing us to remain quite confident about our data.

The first assumption that was implicitly made in our experimental design regards the nature of the equilibrium played by the subjects - namely a mixed-strategy equilibrium. Indeed, the four predictions P1 to P4 we aimed at investigating were obtained at mixedstrategy equilibrium. But the model itself offers multiple equilibria, and we can actually not be sure that our subjects coordinated toward the mixed-strategy equilibrium. Furthermore, CDR (2007)'s theoretical results were obtained under the assumption of symmetry, under which the agents are assumed to consider that any partner of the same type should have the same beliefs as theirs. Obviously, this may not be true in the real world. In our experiment, it may have been the case that some of our subjects considered that their fellow player would have different beliefs and would play a different equilibrium.

Though theoretically valid, we think that this argument can be dismissed using several empirical counter-arguments. First, the qualitative similarity between the theoretical and empirical curves (see Graphs 1 to 4, Section 3 supra) suggests that the experiment succeeded quite well in capturing the qualitative features of the model. Second, even though the subjects did actually not elaborate mixed strategies, the fact that aggregate behaviour roughly coincides with mixed-strategy equilibrium behaviour gives some weight to the as if hypothesis, which has received some support in economics (Friedman and Savage, 1948). By the way, mixed strategies can also be interpreted as the aggregate result of the mix of different pure strategies among the subjects. 
The second assumption we wish to discuss here concerns the categorization of the population. The questionnaire implicitly followed CDR (2007) in assuming that the population of subjects is divided into two classes of agents, namely the SCs and the UCs. As already mentioned in Section 3, we are well aware that these a priori categories are unlikely to exhaust the components of real world population, and that some other behavioural types could be identified (for instance a category of 'unconditional defectors', who always choose to defect; see Neugebauer et al., 2008 for an example of a four-type categorization). Obviously, if some subjects in our experimental study turned out to be of another type, this would prevent us from testing the two-type model under consideration. However, several counter-arguments can be put forward, that minimize the empirical weight of the previous argument. First, in the pilot experiment as well as in the final experiment, none of the subjects showed either disagreement or surprise toward the two-type categorization when filling out the questionnaire. Furthermore, remember that only those subjects whose behaviour was consistent with the behaviour of a SC were retained for data analysis. Maybe they were actually something else, but for our purpose, only their behaviour matters. Besides, the subjects whose behaviour was inconsistent with expected-value maximizing were simply considered as non-SCs. Implicitly, this allowed us to take into account the possibility that some subjects may be neither SCs nor UCs. And to keep the data as unambiguous as possible, these subjects were not included in the final data set.

\subsection{Discussion of results}

\subsubsection{The influence of social determinants}

Even though our experimental design may have introduced some noise in the data, it is unclear how the simplifications we made could be responsible for the high level of cooperation observed among the subjects.

However, such factors as personality as well as social or equity considerations (reviewed in the introduction) may have obviously contributed to our results. For instance, a huge amount of recent literature from both social psychology and economics suggests that, contrary to what basic theory suggests, people do care about the way gains are shared out between them and others (McClintock and Liebrand 1988) and tend to exhibit social preferences such as preference for reciprocity or inequity aversion (see Fehr and Fischbacher 2002 for a survey). Preference for reciprocity entails that actions that are perceived to be kind 
(respectively hostile) will be reciprocated in a kind (respectively hostile) manner (Rabin 1993; Levine 1998; Charness and Rabin 2002). Inequity aversion can be viewed as a kind of altruism: inequity-averse people will try to promote their partner's earnings - provided these earnings remain acceptable as compared to theirs (Fehr and Schmidt 1999; Bolton and Ockenfels 2000). In this framework, even selfish people may be induced to make 'nonselfish' choices. This may contribute to explain why our subjects cooperated much more than predicted.

Demographic as well as socio-cultural determinants may have also played some role in our results. For instance, social identity has been shown to promote cooperative behaviour among in-group members (Dawes et al. 1988; Kollock 1998b; see also Yamagishi and Kiyionari, 2000 and Simpson, 2006 for some recent work on the links between social identity and cooperation), due to 'the shared and mutual perception by in-group members of their interests as interchangeable' (Turner et al. 1987). Since our sample was highly homogeneous as regards age and human capital (all of them were graduate students), as well as social group identity (most of them were students in a French 'Grandes Ecole'), it may be the case that all these factors combined to induce highly cooperative behaviour.

To complete this discussion, we would like to raise another possible explanation for the high level of cooperation observed among our subjects. This explanation has to do with the subjects' attitude toward risk.

\subsubsection{The influence of attitudes towards risk}

In line with most game theoretical models, our theoretical framework assumes that the SCs maximize the expected value of the game. However, our experimental findings suggest that the SC-type individuals might actually (subjectively) deal with outcome and probability in a non linear fashion, thus exhibit somewhat more sophisticated preferences under risk than usually assumed in game theory.

Intuitively, the basic uncertainty that is associated with the partner's behaviour (Sabater-Grande and Georgantzis, 2002) makes it natural to consider interactive situations as a kind of risky situation - involving risky payoffs with a probability distribution over the payoffs. In this perspective, the decision to cooperate (or trust one's partner) may be viewed by the subject as equivalent to taking a risky bet (Bohnet and Zeckhauser 2004, Eckel and Wilson 2004). If so, her risk orientation is likely to influence her 'cooperative attitude'. In the game of chicken (and contrary to what happens in the PDG), the cooperative strategy (action 
$c$, see figure 1 supra) appears to be less risky than the non cooperative one (action $d$ ), since it allows to avoid the worst payoff in the worst outcome (bilateral defection). So, in a game of chicken, more risk aversion can be expected to result in more cooperation. In our theoretical framework, the risky nature of the game is all the more obvious since it introduces a second kind of uncertainty through the probabilistic type of the partner. The decision maker has to think about both the type (SC or UC) and the behaviour (cooperation or defection) of her partner. To our knowledge, only a few experimental studies have been trying to explicitly link together attitude toward risk and attitude toward cooperation (Bohnet et al. 2008; Bohnet and Zeckhauser 2004; Brennan et al. 2007; Dolbear and Lave 1966; Eckel and Wilson 2004; Sabater-Grande et Georgantzis 2002; Lönnqvist et al. 2009). Unfortunately, no conclusive result can be drawn from these quite contradictory studies. Part of the apparent contradiction may come from the fact that attitude toward risk strongly depends on the way it is elicited. For instance, the subjects' degree of risk aversion turns out to be higher when elicited in an interactive context (in a social dilemma for instance) than in an exogenously risky context (through the comparison of lotteries for instance) (Bohnet and Zeckhauser 2004; Lönnqvist et al. 2009). This may obviously confuse the investigation of the connections between attitude toward risk and proneness to cooperation.

Anyway, a huge body of theoretical as well as empirical literature in the field of individual decision making under risk strongly confirms that most people are not risk neutral and do not treat outcomes and probabilities linearly when making risky decisions (see for instance Camerer, 1995 or Starmer, 2000 for a survey). Insofar as interactive decisions can be viewed as risky, it does not look implausible that individuals use similar decision rules, and deal with outcomes and probabilities in a non linear fashion, instead of maximizing expectedvalue as postulated by the basic model.

Formally, the SCs' probability of defection, given by $\alpha=\frac{(1-T)}{p}$, can be formulated as an explicit function of both the structure of the payoffs and the proportion $p$ of SCs in the population (see Result (i), Section 2, supra). The fact that the probability $\alpha$ (empirically given by the frequency of defective choices) be much lower than theoretically expected can be imputed to either some overweighting of the probability $p$ of meeting a SC, or undervaluation of $(1-T)$, or even both.

For instance, reading graph 1 horizontally shows that the rate of cooperative choices of 0.3 that should be observed for $p=0.7$ is actually observed for $p=0.45$. This suggests that 
subjects behave as if they considered $p$ as much higher than it actually is (they cooperate much more than they theoretically should), i.e. as if they overweighted it.

Similarly, assuming that the subjects deal with outcomes through some utility function $u$ might help understand why they behave as they do when $H$ varies. The gap between observed and predicted behaviour is especially wide (reaching 50 points), and the difference between the two increases then decreases as $H$ grows. Since the impact of $H$ on behaviour is examined for a given proportion of SCs, which is kept constant throughout the questions ( $p=$ 0.75), subjective probability weighting alone cannot account for the data. A more plausible explanation is that the subjects do not value their defection gain as much as they should, had they been expected-value maximizers. For instance, reading graph 3 horizontally shows that the level of defection observed when $H=150 €$ (resp. $H=190$ ) corresponds to what was expected for $H=120 €$ (resp. $H=130 €$ ). This suggests that the subjects behave as if they systematically and significantly (by $60 €$ in the most extreme case) undervalued either the unilateral defection gain $H$ (i.e. $u(H)<H$ ) or the difference between $H$ and the other elements of the matrix (being held constant). Such undervaluation of the defection gain may help understand why the subjects keep on cooperating while they no longer should.

Similarly, since the impact of $L$ on behaviour is examined for a given proportion of SCs (namely $p=0.75$ ), most of the large and varying gap between predicted and observed behaviour cannot be interpreted in terms of probability weighting alone. Reading graph 4 horizontally shows that, for instance, the level of cooperation obtained for $L=50 €$ is identical to the theoretically predicted one for $L=85 €$. This suggests that the subjects behave as if they systematically and significantly (by $35 €$ in the most extreme case) overvalued either the unilateral cooperation gain $L$ (i.e. $u(L)>L$ ) or the difference between $L$ and the other elements of the matrix (being held constant). Such overvaluation of the cooperation gain may help understand why the subjects begin to cooperate while they should keep on defecting.

\section{Conclusion}

The experimental study described in this paper aimed at investigating whether, and to what extent, the structure of the population and the structure of the payoffs are likely to affect cooperative behaviour in a game of chicken - problems rarely studied in the experimental economics literature. For this purpose, we used the theoretical framework developed by Cabon-Dhersin and Ramani (2007), which considered a population with two types of agents, 
depending on their general attitude toward cooperation. We then confronted the main two theoretical predictions of this model with actual experimental behaviour. The first (and rather counterintuitive) prediction is that rational or self-interested individuals tend to cooperate more as their proportion in the population increases. The second prediction is that the level of cooperation is a decreasing (respectively increasing) function of the unilateral defection (respectively cooperation) gain. From a qualitative point of view, both predictions appear to be well supported by our data.

Nevertheless, our data show a much higher level of cooperation than theoretically expected. Although usual psychological, sociological and cognitive explanations should obviously not be excluded, our data suggest that attitudes towards risk, and more specifically the way individuals subjectively deal with outcomes and probabilities, may have also contributed to this strikingly high level of cooperation. Game theory usually assumes expected-value maximizing preferences. Contemplating the possibility that individuals may maximize their expected utility, or even some more sophisticated objective functions (as in 'Prospect Theory', Kahneman and Tversky 1979; Tversky and Kahneman 1992 for instance), may help account for the gap between standard theoretical predictions and observed behaviour.

Unfortunately, our data do not allow us to ensure that the subjects actually dealt with outcomes and probabilities the way we suggest. In this respect, a future track for research would be to build an extension of CDR (2007), in which agents are endowed with preferences that allow them to deal with both outcomes and probabilities in a non-linear fashion. In such an extended framework, perhaps some new predictions could be drawn and confronted with experimental behaviour in a more systematic manner. 


\section{ACKNOWLEDGMENTS}

We are grateful to Edi Karni and Shyama Ramani, as well as to an anonymous referee, for their highly valuable comments and suggestions. The financial support from the Agence Nationale de la Recherche (ANR) for the project "The Dynamics of Nanosciences and Nanotechnologies: perspectives from economics and sociology" is gratefully acknowledged.

\section{REFERENCES}

Baron, J. (2008). Social Dilemmas: Cooperation versus Defection. in Thinking and Deciding. Cambridge, UK: Cambridge University Press ( $4^{\text {th }}$ Edition).

Berg, J., Dickhaut, J. W., McCabe, K. A. (1995). Trust, Reciprocity and Social History. Games and Economic Behavior, 10, 122-142.

Bohnet, I., Greig, F., Herrmann, B., Zeckhauser, R. (2008). Betrayal Aversion: Evidence from Brazil, China, Oman, Switzerland, Turkey and the United States. American Economic Review, 98, 294-310.

Bohnet, I., Zeckhauser, R. (2004). Trust, Risk and Betrayal. Journal of Economic Behavior and Organization, 55, 467-484.

Bolton, G., Ockenfels, A. (2000). A Theory of Equity, Reciprocity and Competition. American Economic Review, 90, 166-193.

Boone, C., de Brabander, B., van Witteloostuijn, A. (1999). The Impact of Personality on Behavior in Five Prisoner's Dilemma Games. Journal of Economic Psychology, 20, 343-377. Boone, C., Witteloostuijn, A. (1999). Competitive and Opportunistic Behavior in a Prisoner's Dilemma Game: Experimental Evidence on the Impact of Culture and Education. Scandinavian Journal of Management, 15, 333-350.

Brennan, G., Gonzalez, L. G., Güth, W., Levati, M. V. (2008). Attitudes toward Private and Collective Risk in Individual and Strategic Choice Situations. Journal of Economic Behavior and Organization, 67, 253-262.

Cabon-Dhersin, M.-L., Ramani, S. V. (2007). Opportunism, Trust and Cooperation: A Game Theoretic Approach with Heterogeneous Agents. Rationality and Society, 79, 203-228.

Camerer, C. (2003). Behavioral Game Theory: Experimental Studies of Strategic Interaction. Princeton: Princeton University Press.

Camerer, C. (1995). Individual Decision Making. in Handbook of Experimental Economics. J. Kagel and A. E. Roth. (Eds). Princeton: Princeton U. Press. 
Carraro, C., Siniscalco, D. (1993). Strategies for the International Protection of the Environment. Journal of public Economics, 52, 309-328.

Charness, G., Rabin, M. (2002). Understanding Social Preferences with Simple Tests. Quarterly Journal of Economics, 117, 817-869.

Dawes, R. M., Thaler R. H. (1988). Cooperation. Journal of Economic Perspectives 2(3), 187-197.

Dawes, R. M., Van de Kragt, A. J., Orbell, J. M. (1988). Not Me or Thee but We: The importance of Group Identity in Eliciting Cooperation in Dilemma Situations: Experimental Manipulations. Acta Psychologica, 68, 83-97.

Dolbear, F. T., Lave, L.B. (1966). Risk Orientation as a Predictor in the Prisoner's Dilemma. Journal of Conflict Resolution, 10, 506-515.

Eckel, C.C., Wilson, R. K. (2004). Is Trust a Risky Decision?. Journal of Economic Behavior and Organization, 55, 447-465.

Erlei, M. (2008). Heterogeneous social preferences. Journal of Economic Behavior and Organization, 65, 436-457.

Falk, A., Fischbacher, U. (2006). A Theory of Reciprocity. Games and Economic Behavior, 54, 293-315.

Fehr, E., Fischbacher, U. (2002). Why Social Preferences Matter - The Impact of Non-Selfish Motives on Competition, Cooperation and Incentives. Economic Journal, 112 (478), C1-C33.

Fehr, E., Gächter, S. (2000). Fairness and Retaliation: The Economics of Reciprocity. Journal of Economic Perspectives, 14, 159-181.

Fehr, E., Schmidt, K. (1999). A theory of Fairness, Competition, and Cooperation. Quarterly Journal of Economics, 3, 817-868.

Friedman, M., Savage, L. J. (1948). The Utility Analysis of Choices Involving Risk. Journal of Political Economy, 56, 279-304.

Gächter, S., Herrmann, B., Thöni, C. (2004). Trust, Voluntary Cooperation, and SocioEconomic Background: Survey and Experimental Evidence. Journal of Economic Behavior and Organization, 55, 505-531.

Goeree, J.K., Holt, C. A. (2001). Ten Little Treasures of Game Theory and Ten Intuitive Contradictions. American Economic Review, 91, 1402-1422.

Güth, W., Ockenfels, P., Wendel, M. (1997). Cooperation Based on Trust: An Experimental Investigation. Journal of Economic Psychology, 18, 18-43.

Hardin, R. (1971). Collective Action as an Agreeable N-Prisoners' Dilemma. Science, 16, $472-481$. 
Kollock, P. (1998a). Social Dilemmas: The Anatomy of Cooperation. Annual Review of Sociology, 24, 183-214.

Kollock, P. (1998b). Transforming Social Dilemmas: Group Identity and Cooperation. In P. Danielson (Ed.), Modelling Rational and Moral Agents (pp. 186-210), Oxford: Oxford University Press .

Kahneman, D., Tversky, A. (1979). Prospect theory: An Analysis of Decisions under Risk. Econometrica, 47, 313-327.

Levine, D. (1998). Modeling Altruism and Spitefulness in Experiments. Review of Economic Dynamics, 1, 593-622.

Lönnqvist, J.-E., Verkasalo, M., Walkowitz, G., Wichardt, P. C. (2009) How to Measure Individual Risk Taking? An Experimental Inquiry. University of Helsinky, Finland and University of Bonn, Germany.

McClintock, C.G., Liebrand, W. B. G. (1988). Role of Interdependence Structure, Individual Value Orientation, and Another's Strategy in Social Decision Making: A transformational Analysis. Journal of Personality and Social Psychology, 55, 396-409.

Neugebauer, T., Poulsen, A., Schram, A. (2008). Fairness and Reciprocity in the Hawk-Dove Game. Journal of Economic Behavior and Organization, 66(2), 243-250.

Poppe, M. (2005). The Specificity of Social Dilemma Situations. Journal of Economic Psychology, 26, 431-441.

Rabin, M. (1993). Incorporating Fairness into Game Theory and Economics. American Economic Review, 83, 1281-1302.

Ridley, M. (1997). The Origins of Virtue: Human Instincts and the Evolution of Cooperation. New York: Viking Penguin.

Sabater-Grande, G., Georgantzis, G. (2002). Accounting for Risk Aversion in Repeated Prisoners' Dilemma Games: An Experimental Test. Journal of Economic Behavior and Organization, 48, 37-50.

Sherman, R. (1969). Risk Attitude and Cost Variability in a Capacity Choice Experiment. Review of Economic Studies, 36, 453-466.

Simpson, B. (2006). Social Identity and Cooperation in Social Dilemmas. Rationality and Society, 18, 443-470.

Snyder, G. H. (1971). "Prisoner's Dilemma" and "Chicken" Models in International Politics. International Studies Quarterly, 15, 66-103.

Starmer, C. (1999). Experiments in Economics: Should We Trust the Dismal Scientists in White Coats?. Journal of Economic Methodology, 6(1), 1-30. 
Starmer, C. (2000). Developments in Non-Expected Utility Theory: The Hunt for a Descriptive Theory of Choice Under Risk. Journal of Economic Literature, 38, 332-382.

Stone, R. W. (2001). The Use and Abuse of Game Theory in International Relations: The Theory of Moves. Journal of Conflict Resolution, 45, 216-244.

Thogersen, J. (2008). Social Norms and Cooperation in Real-Life Social Dilemmas. Journal of Economic Psychology, 29(4), 458-472.

Turner, J. C., Hogg, M. A., Oakes, P.J., Reicher, S. D., \& Weetherell, M. S. (1987). Rediscovering the Social Group: A self-Categorization Theory. Oxford: Basil Blackwell.

Tversky, A., Kahneman, D. (1992). Advances in Prospect Theory: Cumulative Representation of Uncertainty. Journal of Risk and Uncertainty, 5, 297-323.

Wagenaar, W. A., Keren, G. B., Lichtenstein, S. (1988). Islanders and Hostages: Deep and Surface Structures of Decision Problems. Acta Psychologica, 67, 175-188.

Ward, H. (1993). Game Theory and the Politics of the Global Commons. Journal of Conflict Resolution, 37, 203-235.

Wit, A. P., Wilke, H. A. M. (1992). The Effect of Social Categorization on Cooperation in Three Types of Social Dilemmas. Journal of Economic Psychology, 13, 135-151.

Yamagishi, T., Kiyonari, T. (2000). The Group as the Container of Generalized Reciprocity. Social Psychology Quarterly, 63, 116-32. 
You are facing a partner. You both have two available options: play Red or play Blue. You do not know what your partner is going to do, but your gain depends on both your own choice and hers.

The matrix below gives the different choices for you and your partner, as well as the corresponding gains. Your gains are in bold:

\begin{tabular}{|c|c|c|c|}
\hline & & \multicolumn{2}{|c|}{ The choice of my partner } \\
\hline & & Red & Blue \\
\hline \multirow[t]{2}{*}{ My choice } & Red & $100 € ; 100 €$ & $70 € ; 120 €$ \\
\hline & Blue & $120 € ; 70 €$ & $\mathbf{5 0} € ; 50 €$ \\
\hline
\end{tabular}

\section{Question 1.1 :}

You have 100\% chances to meet a partner of type (b) who plays either Red or Blue, depending on her potential gains as well as on what she thinks you are going to play.

Which colour do you choose?

$\square$ Red

$\square$ Blue

\section{Question 1.2 :}

You have $\mathbf{1 0 0 \%}$ chances to meet a partner of type (a) who always plays Red.

Which colour do you choose?

\section{$\square$ Red}

$\square$ Blue

\section{Question 1.3 :}

We are now in the general case. Your partner has:

- $\mathbf{X \%}$ chances to be of type (a), in which case she always plays Red, and

- (100-X)\% chances to be of type (b), in which case she plays either Red or Blue, depending on her potential gains as well as on what she thinks you are going to play.

Which colour do you choose for the different values of $X$ that are given in the table below? Just tick the appropriate box (Red or Blue) for each of these values.

\begin{tabular}{|c|c|c|c|c|c|c|c|c|c|c|}
\hline $\begin{array}{c}X \% \text { chances to meet } a \\
\text { partner of type }(a)\end{array}$ & $\mathbf{0} \%$ & $\mathbf{1 0 \%}$ & $\begin{array}{c}25 \% \\
(\mathrm{or}\end{array}$ & $\begin{array}{c}33 \% \\
(\mathrm{or}\end{array}$ & $\mathbf{4 0 \%}$ & $\mathbf{5 0 \%}$ & $\mathbf{6 0 \%}$ & $\begin{array}{c}66 \% \\
(\mathrm{or}\end{array}$ & $\begin{array}{c}\mathbf{7 5 \%} \\
(\mathrm{or}\end{array}$ & $\begin{array}{c}\mathbf{9 0 \%} \\
\mathbf{1 0 0 \%}\end{array}$ \\
\hline
\end{tabular}




\begin{tabular}{|c|c|c|c|c|c|c|c|c|c|c|c|}
\hline $\begin{array}{c}\text { (who always plays Red) } \\
\text { Your choice } \\
\downarrow\end{array}$ & & & 1/4) & $\mathbf{1 / 3})$ & & & & 2/3) & 3/4) & & \\
\hline Red & & & & & & & & & & & \\
\hline Blue & & & & & & & & & & \\
\hline
\end{tabular}

Question 1.1.

Question 1.2. 


\section{Appendix B: The performance-based payment procedure}

At the beginning of the session, the subjects were given full information about the following payment procedure. They were given several examples of how it works, so that they could be aware of their interest to give sincere and well-considered answers when filling out the questionnaire.

The subjects were informed that two of them (the 'winners' in the following) would be selected at random among the participants and that, for each of the 'winners', a choice situation would then be selected at random and played out for real against her partner in the game. Her final payment would thus depend on both her own choice and the choice made by her partner in the situation/scenario under consideration. Before filling out the folder, the subjects were thus made aware that it was in their interest to carefully take into account the behavioural characteristics of their partner when making their decision in each choice situation (that is, to carefully take into account probabilistic information, provided in each scenario, regarding the distribution of behavioural types in the population). They were made aware that, when implementing the final payment, the uncertainty as regards the type of their partner in the randomly selected choice situation would be solved by drawing it at random using the proportions given in the scenario under consideration.

Suppose that, in the scenario considered, $75 \%$ of the population is supposed to be of SC-type. Then, each 'winner' was invited to pick up a token among four tokens numbered from 1 to 4 and informed that, if she picked up the token numbered 1 (resp. numbered from 2 to 4), it would mean that her partner was a UC (resp. a SC). If the partner turned out to be a UC (who always cooperates), the gain of the winner would be determined at the intersection between her own previous choice to the question under consideration and the cooperative choice of her UC-type partner. If the partner turned out to be a SC, the gain of the winner would be determined at the intersection between her own previous choice to the question under consideration and the choice made by a real SC-type subject, picked up at random among all the SC-type subjects in the experiment. Since all the participants had been attributed a number at the beginning of the experiment, a number was selected at random among all the numbers corresponding to those participants whose behaviour was SCcompatible (that is, who had chosen not to cooperate on Question 1.2; see Section 3 supra). The choice of this SC-type subject (remaining anonymous to the 'winner') was then confronted to the 'winner's choice to determine her final payment. 